\title{
Editorial
}

\section{Cardiovascular Professionals for Services Provision in Nepal}

\author{
Koju R
}

Dhulikhel Hospital - Kathmandu University School of Medical Sciences, Dhulikhel, Kavre

Cardiovascular Diseases (CVDs) is increasing globally, accounting for $33 \%$ of deaths worldwide. It is becoming an epidemic in low-income countries and is projected to increase substantially over the next two decades. World Health Organization (WHO) predicted that between 1990 to 2020 , CVDs will increase by $55 \%$ in the world. Nepal, one of the low income countries, is also facing high burden of CVDs affecting elderly to young, men and women, rich and poor $(\mathrm{WHO}, 2011)$. It leads to one in five deaths in developing countries and additionally, $25 \%$ of all deaths is attributable to cardiovascular causes in Nepal.

The number of patients with heart disease and stroke attending hospitals, health facilities are growing each day. This situation is not only prevalent in urban areas but is also increasing in rural and semi-urban areas. Literature review shows that the conditions and risk factors such as hypertension, diabetes mellitus, smoking, obesity, dyslipidemia are reported to be increasing in various population based cross sectional studies in rural and urban areas (eg. Dharan, Kathmandu, Dhulikhel) across the country.

Nepal is facing an epidemiological transition creating new challenges in public health system. Although, Nepal has experienced steady improvement in health outcomes and impacts during the last two decades (NHSIP, 2010), the burden of communicable diseases is still high (NHRC, 2010). A hospital-based study found that out of total admitted patients, $36.5 \%$ suffered from non-communicable diseases. This shows that Nepal has double burden - communicable as well as non-communicable disease.

Though, the number of risk factors, morbidity and mortality due to heart diseases and stroke are rising, the awareness, diagnosis, treatment, and prevention programs for these diseases are limited. The problem of CVDs is getting into an iceberg phenomenon: huge portion of population having cardiac risk factors are unaware and undetected. Majority of the patients whor are diagnosed is also incidentally. General physician, medical doctors, paramedics and few cardiologists and cardiothoracic surgeons are involved in the care of these diagnosed cases.

Even though the burden of cardiovascular problems has been increasing, the required infrastructure for treatment, required number of cardiovascular professionals have not been developed and trained proportionately within the country. Few number of physicians and surgeons having fellowship and special training in cardiology and cardiothoracic surgery have been providing the cardiac services since the beginning. They were the pioneers starting cardiovascular services in the country. For last few years, cardiologists and cardiac surgeons with the degrees like, DM, MD Cardiology, MS, MCh Cardiovascular and Thoracic surgery and Fellowships are growing and providing specialized care with interventional and surgical services.

The population based study showed the prevalence of CVD is $5.7 \%$ which will be about 1.6 million people at one time. The available Cardiovascular professional specialist within Nepal, according to the registration of professional body, Cardiac Society of Nepal is 92. Among these specialists, majority are working in Kathmandu, the capital city, and few in other major cities like Pokhara, Biratnagar, Chitawan, Birganj and Dharan. The proportion of cardiovascular specialists to the cardiac patients in Kathmandu is about 1: 2,500 and the proportion of cardiovascular specialists to the general population is about $1: 45,000$. This is worse in other cities and there is virtually none in rural areas. 
There is severe scarcity of cardiac doctor in each level of health facilities from zonal hospitals to primary level. This fact signifies the challenge in getting proper management of CVDs. Beside the Cardiac Physicians and Surgeons, the specialized nurses, public health exports, anesthesiologists, perfusionists, imaging specialists are also required to optimize the quality services.

Most of the cardiovascular specialists in recent years, (DM, MD Cardiology MS, MCh cardiothoracic surgeon) have been trained from countries such as India, Bangladesh, China. Significant number of other physicians has fellowship from different colleges and institutions such as Russia, Asia Pacific Society of Cardiology, American College of Cardiology, European society of Cardiology etc. Even though the specialized training in cardiology is of great demand, there was no specific course within Nepal few years back. With the establishment of few specialized cardiovascular centers in different hospitals and medical colleges (e.g. Bir Hospital, Shaheed Gangalal National Heart Centre, Manamohan Cardio Thoracic Centre, Norvic
International Hospital, Dhulikhel Hospital, BPIKHS: Dharan, College of Medical Science, Bharatpur and others), DM Cardiology, MCh Cardiothoracic Surgery course have been initiated recently. National Academy of Medical Sciences, Bir Hospital, Tribhuvan University, Institute of Medicine and Kathmandu University School of Medical Sciences have started MCh Cardiothoracic Surgery and DM Cardiology since 2008. The number of seats for this course is limited, although the need of human resources in cardiovascular services is huge.

The development of academic and training programs related to cardiovascular diseases in Nepal in the last few years has been encouraging, although by no means sufficient. There needs to be aggressive expansion of cardiac care facilities, training and research opportunities supported by strong and effective plans and policies. The state as well as public and private institutions in all levels and capacity should gear up for this, not as rivals but as partners dedicated for a common cause. 